\title{
Erratum to: Impact of correlated inputs to neurons: modeling observations from in vivo intracellular recordings
}

\author{
Man Yi Yim • Arvind Kumar • Ad Aertsen • \\ Stefan Rotter
}

Published online: 18 June 2014

(C) Springer Science+Business Media New York 2014

\section{Erratum to: J Comput Neurosci}

DOI 10.1007/s10827-014-0502-z

In the original version of this article, the list of acknowledgements was incomplete.

The complete list of acknowledgements is given here:

The present work was supported by the German Federal Ministry of Education and Research (BMBF grant no. $01 \mathrm{GQ0420}$ to "BCCN Freiburg", BMBF grant no. 01GW0730 "Impulse Control"), the EU (INTERREG-V Grant to Neurex: TriNeuron) and the BrainLinks-BrainTools Cluster of Excellence funded by the German Research Foundation (DFG, grant no. EXC 1086).

The online version of the original article can be found at http://dx.doi.org/ 10.1007/s10827-014-0502-z.

M. Y. Yim

Department of Mathematics, University of Hong Kong, Pokfulam

Road, Hong Kong, China

A. Kumar $\cdot$ A. Aertsen $\cdot$ S. Rotter $(\bowtie)$

Bernstein Center Freiburg and Faculty of Biology, University of

Freiburg, Hansastr. 9a, 79104 Freiburg, Germany

e-mail: stefan.rotter@biologie.uni-freiburg.de 